\title{
Recovery of Genes Epigenetically Altered by the Histone Deacetylase Inhibitor Scriptaid and Demethylating Agent 5-Azacytidine in Human Leukemia Cells
}

\author{
Eunkyung Park ${ }^{1,2}$, Eunhyung Jeon ${ }^{1}$, In Ho Kim ${ }^{1,3}$ \\ and Seonyang Park ${ }^{1,3 *}$
}

${ }^{1}$ Diagnostic DNA Chip Center, Seoul National University College of Medicine, Seoul, Korea, ${ }^{2}$ Department of Internal Medicine, Chung-Ang University College of Medicine, Seoul 156-755, Korea, ${ }^{3}$ Department of Internal Medicine, Seoul National University College of Medicine, Seoul 110-744, Korea

\begin{abstract}
Histone deacetylation and demethylation are epigenetic mechanisms implicated in cancer. Studies regarding the role of modulation of gene expression utilizing the histone deacetylase inhibitor scriptaid and the demethylating agent 5-azacytidine in HL-60 leukemia cells have been limited. We studied the possibility of recovering epigenetically silenced genes by scriptaid and 5-azacytidine in human leukemia cells by DNA microarray analysis. The first group was leukemia cells that were cultured with 5-azacytidine. The second group was cultured with scriptaid. The other group was cultured with both agents. Two hundred seventy newly developed genes were expressed after the combination of 5-azacytidine and scriptaid. Twenty-nine genes were unchanged after the combination treatment of 5-azacytidine and scriptaid. Among the 270 genes, 13 genes were differed significantly from the control. HPGD, CPA3, CEACAM6, LOC653907, ETS1, RAB37, PMP22, FST, FOXC1, and CCL2 were up-regulated, and IGLL3, IGLL1, and $A S S 1$ were down-regulated. Eleven genes associated with oncogenesis were found among the differentially expressed genes: ETS1, ASCL2, BTG2, BTG1, SLAMF6, CDKN2D, RRAS, RET, GIPC1, MAGEB, and RGL4. We report the results of our leukemia cell microarray profiles after epigenetic combination therapy with the hope that they are the starting point of selectively targeted epigenetic therapy.
\end{abstract}

Keywords: deacetylation, demethylation, epigenetic, leukemia, microarray

*Corresponding author: E-mail seonpark@plaza.snu.ac.kr Tel +82-2-2072-3347, Fax +82-2-744-6075

Accepted 2 December 2010

\section{Introduction}

Chromatin remodeling is important in gene expression, DNA repair, and replication (Bishton et al., 2007). Methylation of promotor regions of tumor suppressor genes and histone deacetylation lead to gene silencing (Bishton et al., 2007). Epigenetic modification with histone deacetylase inhibitors and hypomethylating agents allow upregulation of proapoptotic genes and downregulation of antiapoptotic genes, and show significant single-agent anticancer activity (Bishton et al., 2007; Dalgard et al., 2008). Combinations of these drugs are being employed in clinical trials to target multiple biological pathways (Bishton et al., 2007). Preclinical studies of combinations of these agents are ongoing and demonstrate synergy in many hematological cancers (Bishton et al., 2007). But, the exact target is still ambiguous and can not be controlled precisely. We report the results of our leukemia cell microarray profiles after epigenetic combination therapy.

There are several reasons why both demethylating agents and histone deacetylase inhibitors (HDACis) are excellent candidates for examination as part of combination strategies (Bishton et al., 2007). First, these agents have considerable single-agent activity in hematological malignancies; this activity is probably related to the modification of epigenetic changes, leading to re-expression of aberrantly silenced tumor suppressor genes (Bishton et al., 2007). However, the relatively low response of single agents in myelodysplastic syndromes and acute myeloid leukemia demonstrates that there are numerous cellular 'escape mechanisms' that allow the cell to avoid cell death (Bishton et al., 2007). Combination approaches with demethylating agents and HDACis that are targeted at the epigenome may overcome some of this resistance. Second, a multitarget hit strategy seems to benefit cancer therapy. As we dissect critical cell cycle, proliferation, apoptosis, angiogenesis, and signaling pathways, we can develop molecules to target them (Bishton et al., 2007). It is clear that in addition to the effect on histone acetylation, non-histone targets are critical for the effects of these drugs. Such targets for hyperacetylation include p53, $\alpha$-tubulin, HIF-1 $\alpha$, and HSP90 (Bali et al., 2005; Huang et al., 2002; Kawaguchi et al., 2003; Qian et al., 2006). Finally, the good toxicity profile of these agents make them suitable for combina- 
Table 1. Drug combination density options to determine the effect of the combination of 5-azacytidine and scriptaid

\begin{tabular}{lccccc}
\hline Solution $(2 \mathrm{ml})$ & Control & A $2.5 \mu l+S 2.5 \mu l$ & A $5 \mu|+S 5 \mu|$ & A $10 \mu|+S 10 \mu|$ & A $20 \mu l+S 20 \mu l$ \\
\hline 1st set (O.D.) & 2.02 & 0.7 & 0.3 & 0.24 & 0.25 \\
2nd set (O.D.) & 2.27 & 0.51 & 0.38 & 0.27 & 0.27 \\
3rd set (O.D.) & 2.56 & 0.52 & 0.4 & 0.37 & 0.24 \\
\hline
\end{tabular}

O.D. stands for optical density. A stands for 5-azacytidine, and S stands for scriptaid.

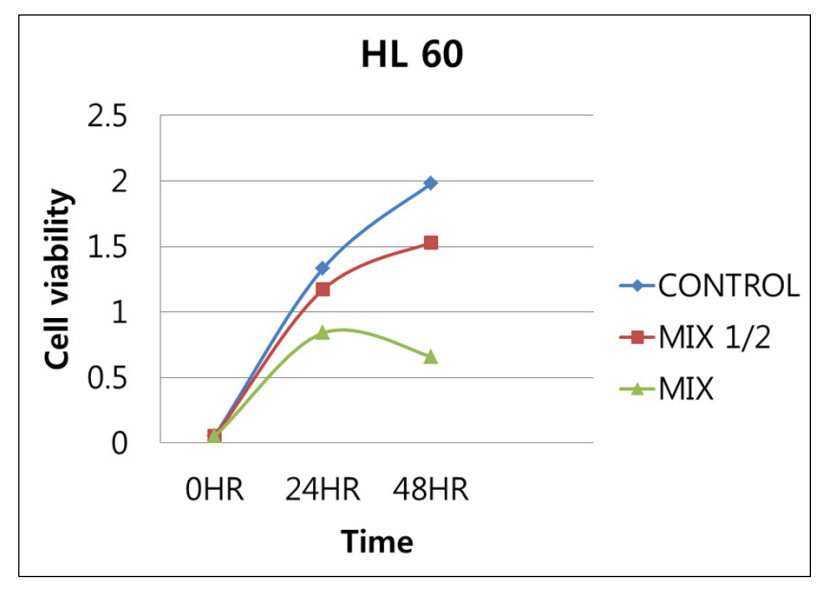

Fig. 1. HL-60 cell viability curve after treatment with 5 -azacytidine and scriptaid. Mix $1 / 2$ group stands for the half-dose of each drug combination compared with the MIX group.

tion therapy (Rasheed et al., 2007).

\section{Methods}

\section{Cell lines \& Cell culture}

The HL-60 leukemic cell line was obtained from Korean Cell Line Bank (KCLB). Cell lines were cultured at $37^{\circ} \mathrm{C}$ $\left(5 \% \mathrm{CO}_{2}\right)$ in RPMI 1640 medium, supplemented with $10 \%$ fetal calf serum and $1 \%$ penicillin/streptomycin (all purchased from Sigma-Aldrich Co. Ltd. Poole, UK).

\section{Determination of effects of drug combination}

5-azacytidine $(12.25 \mu \mathrm{M})$ and $0.83 \mu \mathrm{M}$ scriptaid were used. A 2-mL solution of control and 5-azacytidine 2.5 $\mu \mathrm{L}$, scriptaid $2.5 \mu \mathrm{L}$ combination regimen were compared (Table 1). Cell viability curves were drawn from the combination-treated cells (Fig. 1).

\section{WST-1 assay}

Cell number and viability were further assessed by using the WST-1 reagent (Roche, Indianapols, USA). This col-

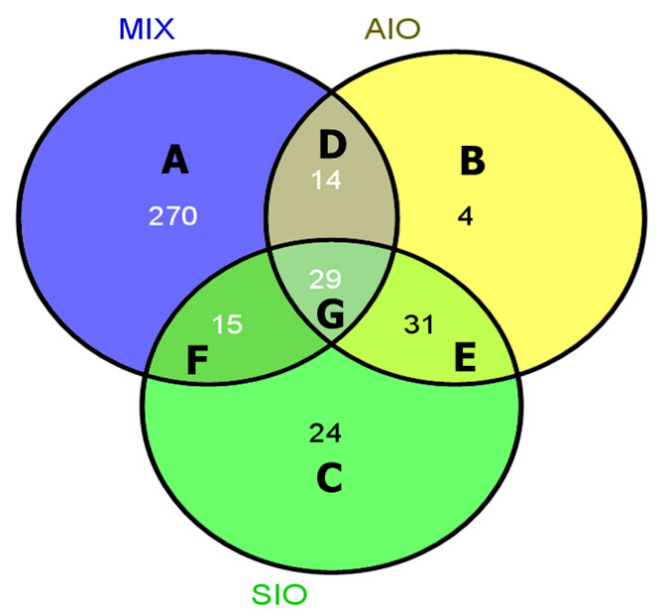

Fig. 2. Expressed gene numbers in leukemia cells after treatment with 5-azacytidine, scriptaid, and the combination regimen (AIO: 5-azacytidine; SIO: Scriptaid; MIX: treatment with both 5-azacytidine and scriptaid).

orimetric assay measures the metabolic activity of viable cells based on cleavage of the tetrazolium salt WST-1 into formazan by mitochondrial dehydrogenase in live cells. Cells under different treatments were plated at $1 \times 10^{4}$ cells $/ 0.32 \mathrm{~cm}^{2}$ in a 96 -well plate and were maintained for $48 \mathrm{hr}$ at $37^{\circ} \mathrm{C}$ in $8.5 \% \mathrm{CO}_{2}$. Absorbance readings were normalized against control wells with medium alone. All experiments were performed independently at least 3 times in triplicate per experimental point.

\section{RNA extraction}

Total RNA was isolated from cell lines using Nucleospin RNA II (Macherey-Nagel, Germany). RNA quality was assessed using a NanoDrop ND-1000 (NanoDrop Technologies, Wilmington, USA) spectrophotometer. Extracted RNA was stored at $-70^{\circ} \mathrm{C}$.

Total RNA yield was quantified by densitometric assessment of both ribosomal bands using the FlourChem Digital Imaging System (Alpha Inotek, Inc., San Leandro, CA, USA). 


\section{Microarray analysis}

The microarray hybridization was performed using the Illumina Gene Expression 48K Chip (Illumina). Data visualization, clustering, and differential analysis were carried out with BeadStudio software (Illumina) and the GeneSpring GX program, 7.3 (Silicon Genetics).

A
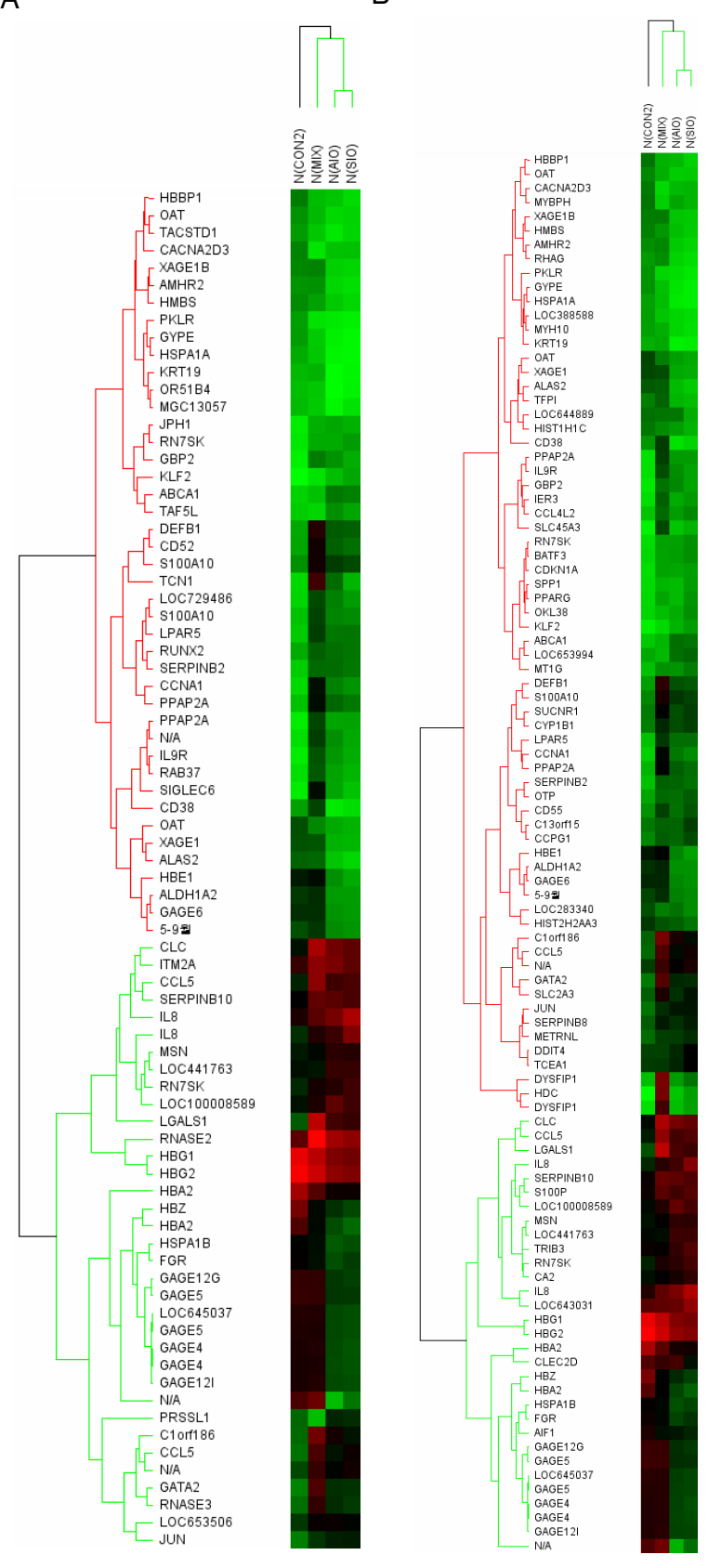

Fig. 3. Gene expression profile of leukemia cells after treatment. (A) 5-Azacytidine, (B) Scriptaid.

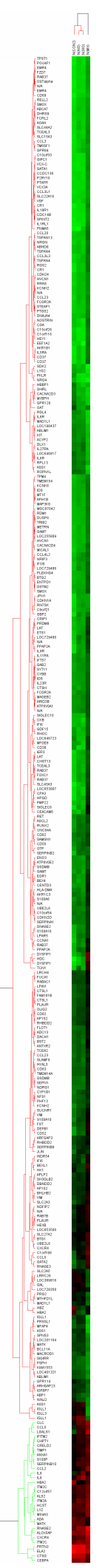

Fig. 4. Gene expression profile of leukemia cells after treatment with combination regimen of 5-azacytidine and scriptaid. 


\section{Results}

\section{Determination of effects of drug combination}

The 5-azacytidine and scriptaid combination regimen-treated leukemia cells were decreased after 48 hours of incubation. The demethylating agent and HDACi combination regimen can be an option to decrease leukemia cells (Fig. 1).

Differentially expressed genes in leukemia cells treated with 5-azacytidine, scriptaid, or the combination regimen

There were 78 expressed genes after treatment with 5-azacytidine (Fig. 2, 3). Ninety-nine genes were expressed after treatment with scriptaid (Fig. 2, 3). Sixty genes overlapped between the 5-azacytidine treatment and scriptaid treatment (Fig. 2).

After treatment with both 5-azacytidine and scriptaid, which is a combination of a demethylating agent and an $\mathrm{HDACi}$, there was a remarkable gene expression profile change (Fig. 4, Table 2). Two hundred seventy newly developed genes were expressed (Fig. 2, Table 2). The gene ontology biological process profile shows that of these 270 genes, 94 genes were associated with signal transduction (34.81\%), 68 genes were associated with immunity and defense $(25.19 \%)$, and 35 genes were associated with developmental processes (12.96\%) (Fig. 4, Table 2). The gene ontology molecular function profile shows that 32 genes were associated with receptors (11.85\%), 23 genes were associated with signaling molecules (8.52\%), and 19 genes were associated with select regulatory molecules (7.04\%) (Fig. 5, Table 2).

We selected 13 genes that showed higher foldchanges after treatment with combination therapy with 5-azacytudune and scriptaid. HPGD, CPA3, CEACAM6, LOC653907, ETS1, RAB37, PMP22, FST, FOXC1, and CCL2 were up-regulated, and IGLL3, IGLL1, and ASS1 were down-regulated (Table 3).

Differentially expressed genes associated with oncogenesis in leukemia cells treated with the 5-azacytidine and scriptaid combination regimen

Of the 270 genes, 11 genes were involved in oncogenesis: ETS1, ASCL2, BTG2, BTG1, SLAMF6, CDKN2D, RRAS, RET, GIPC1, MAGEB, and RGL4 (Fig. 2, Table 4).

Twenty-nine genes remained unchanged after combination treatment with 5-azacytidine and scriptaid (Fig. 2, Table 5). Two genes were involved in oncogenesis: CCNA1 and JUN (Fig. 2, Table 5).
Table 2. Functional annotation of differentially expressed genes in leukemia cells after treatment with combination regimen of 5 -azacytidine and scriptaid

\begin{tabular}{|c|c|c|}
\hline Category & Term & Count (\%) \\
\hline \multirow[t]{27}{*}{ GO BP } & Signal transduction & $94(34.81)$ \\
\hline & Immunity and defense & $68(25.19)$ \\
\hline & Developmental processes & $35(12.96)$ \\
\hline & Protein metabolism and modification & $25(9.26)$ \\
\hline & $\begin{array}{l}\text { Nucleoside, nucleotide and nucleic } \\
\text { acid metabolism }\end{array}$ & $21(7.78)$ \\
\hline & Cell proliferation and differentiation & $20(7.41)$ \\
\hline & Cell cycle & $16(5.93)$ \\
\hline & Transport & $14(5.19)$ \\
\hline & Cell adhesion & $12(4.44)$ \\
\hline & Cell structure and motility & $12(4.44)$ \\
\hline & Lipid, fatty acid and steroid metabolism & $12(4.44)$ \\
\hline & Oncogenesis & $11(4.07)$ \\
\hline & Other metabolism & $9(3.33)$ \\
\hline & Carbohydrate metabolism & $8(2.96)$ \\
\hline & Intracellular protein traffic & $8(2.96)$ \\
\hline & Neuronal activities & $7(2.59)$ \\
\hline & Apoptosis & $7(2.59)$ \\
\hline & Amino acid metabolism & $6(2.22)$ \\
\hline & Sulfur metabolism & $4(1.48)$ \\
\hline & Sensory perception & $3(1.11)$ \\
\hline & Homeostasis & $2(0.74)$ \\
\hline & Miscellaneous & $2(0.74)$ \\
\hline & Muscle contraction & $2(0.74)$ \\
\hline & Nitrogen metabolism & $2(0.74)$ \\
\hline & Coenzyme and prosthetic group metabolism & n $1(0.37)$ \\
\hline & Phosphate metabolism & $1(0.37)$ \\
\hline & Protein targeting and localization & $1(0.37)$ \\
\hline \multirow{24}{*}{ GO MF } & Receptor & $32(11.85)$ \\
\hline & Signaling molecule & $23(8.52)$ \\
\hline & Select regulatory molecule & $19(7.04)$ \\
\hline & Defense/immunity protein & $16(5.93)$ \\
\hline & Transcription factor & $16(5.93)$ \\
\hline & Oxidoreductase & $14(5.19)$ \\
\hline & Nucleic acid binding & $13(4.81)$ \\
\hline & Miscellaneous function & $12(4.44)$ \\
\hline & Cell adhesion molecule & $11(4.07)$ \\
\hline & Hydrolase & $10(3.70)$ \\
\hline & Protease & $10(3.70)$ \\
\hline & Transporter & $9(3.33)$ \\
\hline & Kinase & $7(2.59)$ \\
\hline & Phosphatase & $6(2.22)$ \\
\hline & Transferase & $6(2.22)$ \\
\hline & Ligase & $5(1.85)$ \\
\hline & Cytoskeletal protein & $4(1.48)$ \\
\hline & Membrane traffic protein & $4(1.48)$ \\
\hline & Extracellular matrix & $3(1.11)$ \\
\hline & Lyase & $3(1.11)$ \\
\hline & Synthase and synthetase & $2(0.74)$ \\
\hline & Chaperone & $1(0.37)$ \\
\hline & Ion channel & $1(0.37)$ \\
\hline & Select calcium binding protein & $1(0.37)$ \\
\hline
\end{tabular}

BP: biological process, GO: gene ontology, MF: molecular function. Count indicates the number of genes assigned to the gene ontology term. 


\section{Discussion}

Demethylating agents, such as 5-azacytidine and decitabine, are actively implicated in clinical practice to improve survival and quality of life in myelodysplastic syndrome. Their potential action on acute leukemia is also being studied. HDACis, such as scriptaid and valproic acid, are also useful in decreasing leukemia cells (Chavez-Blanco et al., 2006). But, neither of these single agent makes clinicians satisfied as a cure for acute leukemia. There are a few studies that have combined these two epigenetic therapies to reveal silenced tumor suppressor genes. The dual epigenetic therapy may induce positive or negative genetic alterations in leukemia cells, and the gene expression profile may change a lot after combining the two epigenetic therapies--a demethylating agent and an HDACi. There are little data on the gene expression profiles of dual epigenetic therapy so far.

It is notable that over two times as many genes were newly expressed after treatment with both the demethylating agent and $\mathrm{HDACi}$. The newly expressed genes could work positively or negatively on leukemia cells. So, before applying dual epigenetic therapy to the clinical setting, target gene information may help clinicians to understand the mechanisms of leukemogenesis and epigenetic therapy.

Our results show that the major portion of newly expressed genes after combination treatment with 5-aza- cytidine and scriptaid are associated with signal transduction and immunity. The associated signal trans-
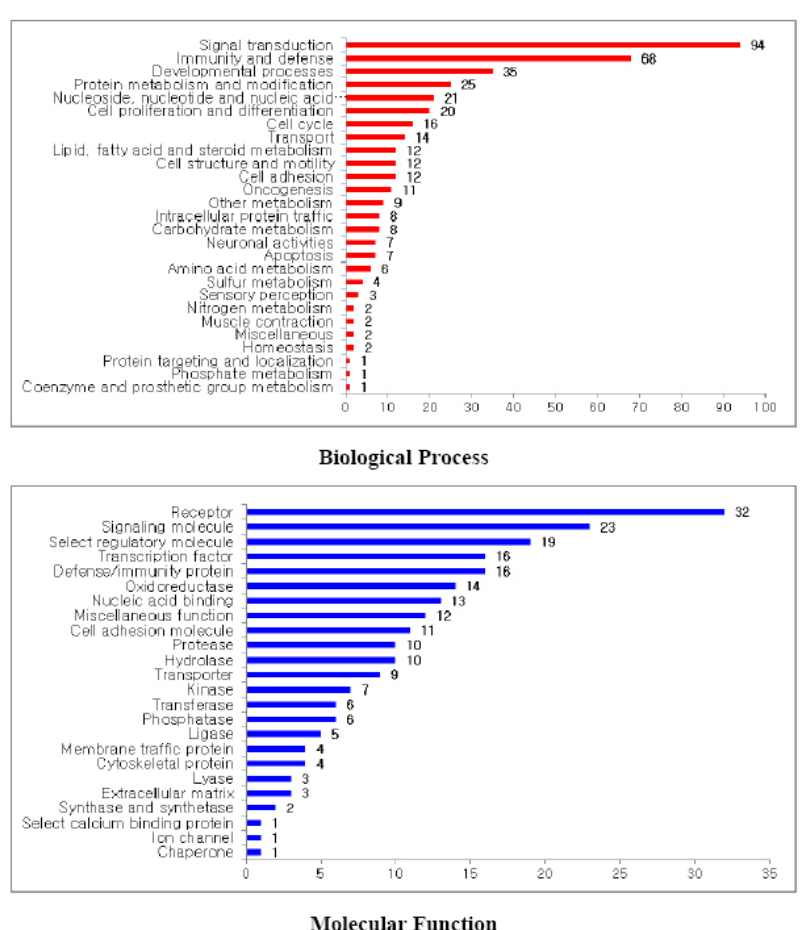

Fig. 5. Gene ontology of gene expression profile of leukemia cells after treatment with combination regimen of 5-azacytidine and scriptaid.

Table 3. Differentially expressed genes in leukemia cells treated with combination regimen with 5-azacytidine and scriptaid

\begin{tabular}{lllll}
\hline \multicolumn{1}{c}{ Gene } & Reporter ID & \multicolumn{1}{c}{ GO BP } & \multicolumn{1}{c}{ GO MF } & Fold \\
\hline HPGD & NM_000860.3 & Oxidoreductase & Lipid, fatty acid and steroid metabolism & 9.24 \\
CPA3 & NM_001870.1 & Protease & Protein metabolism and modification & 8.55 \\
CEACAM6 & NM_002483.3 & Cell adhesion molecule & Signal transduction & 7.44 \\
LOC653907 & XM_936496.2 & Receptor & Immunity and defense & 7.18 \\
ETS1 & NM_005238.2 & Signaling molecule & Nucleoside, nucleotide and nucleic acid metabolism; & 6.76 \\
& & & Oncogenesis & 6.70 \\
RAB37 & NM_175738.3 & Select regulatory molecule & Signal transduction & 6.63 \\
PMP22 & NM_153321.1 & Cytoskeletal protein & Neuronal activities & 5.49 \\
FST & NM_013409.1 & Select regulatory molecule & Homeostasis & 5.29 \\
FOXC1 & NM_001453.1 & Transcription factor & Carbohydrate metabolism; Nucleoside, & \\
& & & nucleotide and nucleic acid metabolism; Signal transduction; & \\
& & & Sensory perception; Developmental processes; Cell cycle; & \\
CCL2 & NM_002982.3 & Signaling molecule & Cell proliferation and differentiation; Cell structure and motility \\
IGLL3 & NM_001013618.1 & Defense/immunity protein & Immunity and defense & 5.17 \\
IGLL1 & NM_020070.2 & Defense/immunity protein & Immunity and defense & -7.10 \\
ASS1 & NM_000050.4 & Ligase & Amino acid metabolism; Nitrogen metabolism & -8.76 \\
\hline BP: & & & -9.02 \\
\hline
\end{tabular}

BP: biological process, GO: gene ontology, MF: molecular function. The fold-change is the ratio of average expression values of combination treatment specimens to those of untreated (control) specimens. A positive and negative score indicates up-regulation and down-regulation in the combination regimen-treated specimens, respectively. 
Table 4. Newly expressed genes associated with oncogenesis in leukemia cells after treatment with the combination regimen of 5-azacytidine and scriptaid

\begin{tabular}{lcc}
\hline Gene & Reporter ID & Fold change \\
\hline ETS1 & NM_005238.2 & 6.76 \\
ASCL2 & NM_005170.2 & 3.18 \\
BTG2 & NM_006763.2 & 3.17 \\
BTG1 & NM_001731.1 & 2.91 \\
SLAMF6 & NM_052931.3 & 2.74 \\
CDKN2D & NM_079421.2 & 2.41 \\
RRAS & NM_006270.3 & 2.39 \\
RET & NM_020630.4 & 2.37 \\
GIPC1 & NM_202468.1 & 2.29 \\
MAGEB2 & NM_002364.3 & 2.22 \\
RGL4 & NM_153615.1 & -2.68 \\
\hline
\end{tabular}

The fold-change is the ratio of average expression values of combination treatment specimens to those of untreated (control) specimens. A positive and negative score indicates up-regulation and down-regulation in the combination regimen-treated specimens, respectively.

duction pathways may be the key to leukemogenesis and a cure for leukemia. But, due to the limitations of cell line data, our results can not generate an answer yet. Further clinical confirmation is needed.

$H P G D$, hydroxyprostaglandin dehydrogenase 15 , encodes a member of the short-chain nonmetalloenzyme alcohol dehydrogenase protein family. The encoded enzyme is responsible for the metabolism of prostaglandins, which function in a variety of physiological and cellular processes, such as inflammation. Mutations in this gene result in primary autosomal recessive hypertrophic osteoarthropathy and cranioosteoarthropathy. A recent study reported the association of a risk of lung cancer with an HPGD SNP (Wang et al., 2010). It is inversely correlated with VEGF expression in colorectal cancer (Lim et al., 2010). HPGD is down-regulated in gastric cancer (Thiel et al., 2009). Reduction of HPGD expression is an independent predictor of poor survival, associated with enhanced cell proliferation in gastric adenocarcinoma (Tatsuwaki et al., 2010). Down- regulation of HPGD contributes to $\mathrm{PGE}_{2}$ accumulation in lung, colon, and pancreatic cancers (Pham et al., 2010). But, $H P G D$ protein level is higher in malignant ovarian tissue samples compared to normal tissue, which does not correlate with the former report (Thill et al., 2010). HPGD is up-regulated in leukemia cells after combination treatment with 5-azacytidine and scriptaid.

$C P A 3$, carboxypeptidase $\mathrm{A} 3$, encodes mast cell carboxypeptidase A (Mc-cpa), which is a highly conserved secretory granule protease. Mc-cpa plays a crucial role in the generation of phenotypically mature mast cells
Table 5. Unchanged genes associated with oncogenesis in leukemia cells after treatment with combination regimen of 5-azacytidine and scriptaid

\begin{tabular}{lcc}
\hline Gene & Reporter ID & Fold change \\
\hline CCNA1 & NM_003914.2 & 10.37 \\
JUN & NM_002228.3 & 2.33 \\
\hline
\end{tabular}

The fold-change is the ratio of average expression values of combination treatment specimens to those of untreated (control) specimens. A positive and negative score indicates up-regulation and down-regulation in the combination regimen-treated specimens, respectively.

(Feyerabend et al., 2005). CPA3 is up-regulated in leukemia cells after combination treatment with 5-azacytidine and scriptaid.

Overexpression of CEACAM6, carcinoembryonic antigen-related cell adhesion molecule 6 , independently predicted poor overall survival in colorectal cancer (Jantscheff et al., 2003). High expression of CEACAM6 mRNA was reported in acute lymphoblastic leukemias (Lasa et al., 2008). CEACAM6 is associated with neutrophil adhesion to the endothelium and angiogenesis (Skubitz and Skubitz 2008). CEACAM6 is a major target gene for Smad3-mediated TGF-beta signaling (Han et al., 2007). There is a report of CEACAM6 overexpression in borderline and invasive ovarian neoplasms (Litkouhi et al., 2008). CEACAM6 is up-regulated in leukemia cells after combination treatment with 5-azacytidine and scriptaid.

LOC653907 is similar to complement component $(3 \mathrm{~b} / 4 \mathrm{~b})$ receptor 1 isoform $\mathrm{F}$ precursor. It is associated with immunity and defense. There are some reports that it is associated with Alzheimer disease or dementia (Bossers et al., 2010). LOC653907 is up-regulated in leukemia cells after combination treatment with 5-azacytidine and scriptaid.

ETS1, erythroblastosis virus E26 oncogene homolog 1 , regulates numerous genes and is involved in stem cell development, cell senescence and death, and tumorigenesis (Russell and Garrett-Sinha, 2010). ETS1 is up-regulated in leukemia cells after combination treatment with 5-azacytidine and scriptaid.

$R A B 37$ is a member of the RAS oncogene family. Rab proteins are low-molecular-mass GTPases that are critical regulators of vesicle trafficking. Frequent down-regulation of $R A B 37$ in lung cancer has been reported (Wu et al., 2009). RAB37 with renal cell cancer association has also been reported (Dobashi et al., 2009). RAB37 is up-regulated in leukemia cells after combination treatment with 5-azacytidine and scriptaid.

PMP22, peripheral myelin protein 22, mediates Schwann cell growth and peripheral myelin compaction. Interleu- 
kin-6 upregulates the expression of PMP22 in cultured rat Schwann cells via a JAK2-dependent pathway (lto et al., 2010). PMP22 is up-regulated in leukemia cells after combination treatment with 5-azacytidine and scriptaid.

$F S T$, follistatin, is a single-chain gonadal protein that specifically inhibits follicle-stimulating hormone release. Overexpression of follistatin in human myoblasts increases their proliferation and differentiation and improves graft success in SCID mice (Benabdallah et al., 2009). FST is up-regulated in leukemia cells after combination treatment with 5-azacytidine and scriptaid.

FOXC1, forkhead box $\mathrm{C} 1$, belongs to the forkhead family of transcription factors, which are characterized by a distinct DNA-binding forkhead domain. The specific function of this gene has not yet been determined; however, it has been shown to play a role in the regulation of embryonic and ocular development. Mutations in this gene cause various glaucoma phenotypes, including primary congenital glaucoma, autosomal dominant iridogoniodysgenesis anomalies, and Axenfeld-Rieger anomalies. There is frequent aberrant DNA methylation of FOXC1 in breast cancer (Muggerud et al., 2010). FOXC1 is up-regulated in leukemia cells after combination treatment with 5-azacytidine and scriptaid.

CCL2, chemokine ligand 2, encodes a monocyte chemoattractant protein. It is up-regulated in leukemia cells after combination treatment with 5-azacytidine and scriptaid.

IGLL3, immunoglobulin lambda-like polypeptide 3, and $I G L L 1$, immunoglobulin lambda-like polypeptide 1, are down-regulated in leukemia cells after combination treatment with 5-azacytidine and scriptaid. ASS1, argininosuccinate synthetase 1 , is also down-regulated in leukemia cells after combination treatment with 5-azacytidine and scriptaid.

Among the 270 genes, 11 genes are involved with oncogenesis. They could be oncogenes or tumor suppressor genes. So, newly expressed tumor suppressor genes, such as $C D K N 2 D$, could function to suppress hematological malignancies. But, newly developed oncogenes could be the cause of secondary malignancies or progression of existing hematological malignancies.

ASCL2, achaete-scute complex homolog 2, controls intestinal stem cell fate (van der Flier et al., 2009). It is a target of Wnt signaling and is upregulated in intestinal neoplasia (Jubb et al., 2006).

BTG2, B-cell translocation gene 2, is a tumor suppressor gene of medulloblastoma (Farioli-Vecchioli et al., 2007). BTG2 plays a pivotal role in maintaining the hematopoietic stem cell compartment and hematopoiesis (Kim et al., 2008). BTG1 is associated with angiogenesis (Iwai et al., 2004).

$S L A M F 6$ is an oncogene associated with B cell adhe- sion (Cannons et al., 2010). CDKN2D, cyclin-dependent kinase inhibitor 2D (p19), and RRAS, Harvey rat sarcoma oncogene, subgroup $R$, are also up-regulated in leukemia cells after combination treatment with 5-azacytidine and scriptaid.

Genetic alterations in the 29 genes that were unchanged after treatment with both the demethylating agent and HDACi might give us solutions to overcome hematological malignancies. There are two genes involved in oncogenesis, CCNA1 and JUN (Fig. 1, Table 4). These two genes could be the target genes to overcome with other chemotherapeutic agents, monoclonal antibodies, or small molecules which cannot be controlled with epigenetic therapy.

There is some evidence that combination strategies with multiple epigenetic agents, such as demethylating agents and HDACis, show synergistic effects and benefits in hematological malignancies (Bishton et al., 2007; Cameron et al., 1999; Chavez-Blanco et al., 2006; Desmond et al., 2007; Galm et al., 2006). But, the lack of long-term follow-up of clinical data and the exact genetic information after treatment with multiple epigenetic therapy makes clinicians hesitate in using epigenetic combination therapy. Demethylating agents, such as 5-azacytidine and decitabine, are nonselective hypomethylating agents that can not selectively target specific chromosomal loci. But, there are synergistic gene expression changes after multiple epigenetic therapy, and information about the target genes could be the weapons to conquer hematological malignancies. There are no previous reports of microarray genetic profiles after treatment with both the demethylating agent 5-azacytidine and the HDACi scriptaid. We report our results on leukemia cell microarray profiles after epigenetic combination therapy with the hope that they are the starting point of selectively targeted epigenetic therapy.

Nonetheless, combination trials will need to be designed carefully, as it is possible that unexpected toxicities may be unearthed. Transient thrombocytopenia is a common dose-dependent toxic effect of HDACis, which may limit our ability to combine these drugs with chemotherapy (Bishton et al., 2007). Large randomized trials will be required to establish that combination approaches are advantageous.

To further our understanding of the targets of these epigenetic agents, careful preclinical studies of mechanisms and pharmacodynamics analysis, incorporated within translational studies on clinical trials, are essential. As our knowledge of the epigenetic control of gene expression expands, novel targets for therapeutic intervention will be identified, and new classes of selectively targeted agents will be developed. 


\section{Acknowledgments}

This study was supported by a grant from the Korean Health 21 R\&D Project, Ministry of Health \& Welfare, Republic of Korea (0405-BC02-0604-0004).

\section{References}

Bali, P., Pranpat, M., Bradner, J., Balasis, M., Fiskus, W., Guo, F., Rocha, K., Kumaraswamy, S., Boyapalle, S., and Atadja, P. (2005). Inhibition of histone deacetylase 6 acetylates and disrupts the chaperone function of heat shock protein 90. J. Biol. Chem. 280, 26729.

Benabdallah, B., Bouchentouf, M., Rousseau, J., and Tremblay, J. (2009). Overexpression of follistatin in human myoblasts increases their proliferation and differentiation, and improves the graft success in SCID mice. Cell Transplantation 18, 709-718.

Bishton, M., Kenealy, M., Johnstone, R., Rasheed, W., and Prince, H. (2007). Epigenetic targets in hematological malignancies: combination therapies with HDACis and demethylating agents. Expert Review of Anticancer Therapy 7, 1439-1449.

Bossers, K., Wirz, K., Meerhoff, G., Essing, A., van Dongen, J., Houba, P., Kruse, C., Verhaagen, J., and Swaab, D. (2010). Concerted changes in transcripts in the prefrontal cortex precede neuropathology in Alzheimer's disease. Brain 133, 3699-3723.

Cameron, E., Bachman, K., Myohanen, S., Herman, J., and Baylin, S. (1999). Synergy of demethylation and histone deacetylase inhibition in the re-expression of genes silenced in cancer. Nature Genet. 21, 103-107.

Cannons, J., Qi, H., Lu, K., Dutta, M., Gomez-Rodriguez, J., Cheng, J., Wakeland, E., Germain, R., and Schwartzberg, P. (2010). Optimal germinal center responses require a multistage $T$ cell: $B$ cell adhesion process involving integrins, SLAM-associated protein, and CD84. Immunity 32, 253-265.

Chavez-Blanco, A., Perez-Plasencia, C., Perez-Cardenas, E., Carrasco-Legleu, C., Rangel-Lopez, E., Segura-Pacheco, B., Taja-Chayeb, L., Trejo-Becerril, C., Gonzalez-Fierro, A., and Candelaria, M. (2006). Antineoplastic effects of the DNA methylation inhibitor hydralazine and the histone deacetylase inhibitor valproic acid in cancer cell lines. Cancer Cell Int. 6, 2.

Dalgard, C., Van Quill, K., and O'Brien, J. (2008). Evaluation of the in vitro and in vivo antitumor activity of histone deacetylase inhibitors for the therapy of retinoblastoma. Clin. Cancer Res. 14, 3113.

Desmond, J., Raynaud, S., Tung, E., Hofmann, W., Haferlach, T., and Koeffler, H. (2007). Discovery of epigenetically silenced genes in acute myeloid leukemias. Leukemia 21, 1026-1034.

Dobashi, S., Katagiri, T., Hirota, E., Ashida, S., Daigo, Y., Shuin, T., Fujioka, T., Miki, T., and Nakamura, Y. (2009). Involvement of TMEM22 overexpression in the growth of renal cell carcinoma cells. Oncol. Reports 21, 305.

Farioli-Vecchioli, S., Tanori, M., Micheli, L., Mancuso, M.,
Leonardi, L., Saran, A., Ciotti, M., Ferretti, E., Gulino, A., and Pazzaglia, S. (2007). Inhibition of medulloblastoma tumorigenesis by the antiproliferative and pro-differentiative gene PC3. The FASEB J. 21, 2215.

Feyerabend, T., Hausser, H., Tietz, A., Blum, C., Hellman, L., Straus, A., Takahashi, H., Morgan, E., Dvorak, A., and Fehling, H. (2005). Loss of histochemical identity in mast cells lacking carboxypeptidase A. Mol. Cell. Biol. 25, 6199.

Galm, O., Herman, J., and Baylin, S. (2006). The fundamental role of epigenetics in hematopoietic malignancies. Blood Rev. 20, 1-13.

Han, S., Kwak, T., Her, K., Cho, Y., Choi, C., Lee, H., Hong, S., Park, Y., Kim, Y., and Kim, T. (2007). CEACAM5 and CEACAM6 are major target genes for Smad3-mediated TGF-â signaling. Oncogene 27, 675683.

Huang, Y., Tan, M., Gosink, M., Wang, $K_{\text {., }}$ and Sun, $Y$. (2002). Histone deacetylase 5 is not a p53 target gene, but its overexpression inhibits tumor cell growth and induces apoptosis. Cancer Res. 62, 2913.

Ito, T., Ikeda, K., Tomita, K., and Yokoyama, S. (2010). Interleukin-6 upregulates the expression of PMP22 in cultured rat Schwann cells via a JAK2-dependent pathway. Neuro. Lett. 472, 104-108.

Iwai, K., Hirata, K., Ishida, T., Takeuchi, S., Hirase, T., Rikitake, Y., Kojima, Y., Inoue, N., Kawashima, S., and Yokoyama, M. (2004). An anti-proliferative gene BTG1 regulates angiogenesis in vitro. Biochem. Biophys. Res. Commun. 316, 628-635.

Jantscheff, P., Terracciano, L., Lowy, A., Glatz-Krieger, K. Grunert, F., Micheel, B., Brummer, J., Laffer, U., Metzger,

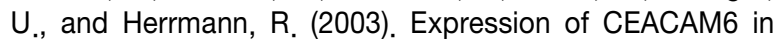
resectable colorectal cancer: a factor of independent prognostic significance. J. Clin. Oncol. 21, 3638.

Jubb, A., Chalasani, S., Frantz, G., Smits, R., Grabsch, H., Kavi, V., Maughan, N., Hillan, K., Quirke, P., and Koeppen, H. (2006). Achaete-scute like 2 (ascl2) is a target of Wnt signalling and is upregulated in intestinal neoplasia. Oncogene 25, 3445-3457.

Kawaguchi, Y., Kovacs, J., McLaurin, A., Vance, J., Ito, A., and Yao, T. (2003). The deacetylase HDAC6 regulates aggresome formation and cell viability in response to misfolded protein stress. Cell 115, 727-738.

Kim, B., Ryu, M., Oh, S., and Lim, I. (2008). TIS21/BTG2 negatively regulates estradiol stimulated expansion of hematopoietic stem cells by derepressing akt phosphorylation and inhibiting mTOR signal transduction. Stem Cells 26, 2339-2348.

Lasa, A., Serrano, E., Carricondo, M., Carnicer, M., Brunet, S., Badell, I., Sierra, J., Aventin, A., and Nomdedeu, J. (2008). High expression of CEACAM6 and CEACAM8 mRNA in acute lymphoblastic leukemias. Ann. Hematol. 87, 205-211.

Lim, S., Cho, H., Lee, T., Choi, C., Min, Y., Kim, S., and Kim, K. (2010). Impacts of Cytosolic Phospholipase A2, 15-Prostaglandin Dehydrogenase, and Cyclooxygenase-2 Expressions on Tumor Progression in Colorectal Cancer. Yonsei Medical J. 51, 692. 
Litkouhi, B., Fleming, E., Welch, W., Berkowitz, R., Birrer, M., and Mok, S. (2008). Overexpression of CEACAM6 in borderline and invasive mucinous ovarian neoplasms. Gynecol. Oncol. 109, 234-239.

Muggerud, A., Ronneberg, J., Warnberg, F., Botling, J., Busato, F., Jovanovic, J., Solvang, H., Bukholm, I., Borresen-Dale, A., and Kristensen, V. (2010). Frequent aberrant DNA methylation of ABCB1, FOXC1, PPP2R2B and PTEN in ductal carcinoma in situ and early invasive breast cancer. Breast Cancer Res, 12, R3.

Pham, H., Chen, M., Li, A., King, J., Angst, E., Dawson, D., Park, J., Reber, H., Hines, O., and Eibl, G. (2010). Loss of 15-hydroxyprostaglandin dehydrogenase increases prostaglandin E2 in pancreatic tumors. Pancreas 39, 332.

Qian, D., Kachhap, S., Collis, S., Verheul, H., Carducci, M., Atadja, P., and Pili, R. (2006). Class II histone deacetylases are associated with $\mathrm{VHL}$-independent regulation of hypoxia-inducible factor 1 alpha. Cancer Res. 66, 8814.

Rasheed, W., Johnstone, R., and Prince H. (2007). Histone deacetylase inhibitors in cancer therapy. Expert Opin. Investig. Drugs 16, 659-678.

Russell, L., and Garrett-Sinha, L. (2010). Transcription factor Ets-1 in cytokine and chemokine gene regulation. Cytokine 51, 217-226.

Skubitz, K., and Skubitz, A. (2008). Interdependency of CEACAM-1,-3,-6, and-8 induced human neutrophil adhesion to endothelial cells. J. Translational Medicine 6, 78.

Tatsuwaki, H., Tanigawa, T., Watanabe, T., Machida, $\mathrm{H}_{\text {., }}$ Okazaki, H., Yamagami, H., Shiba, M., Watanabe, K., Tominaga, K., and Fujiwara, Y. (2010). Reduction of 15 hydroxyprostaglandin dehydrogenase expression is an in- dependent predictor of poor survival associated with enhanced cell proliferation in gastric adenocarcinoma. Cancer Sci. 101, 550-558.

Thiel, A., Ganesan, A., Mrena, J., Junnila, S., Nykanen, A., Hemmes, A., Tai, H., Monni, O., Kokkola, A., and Haglund, C. (2009). 15-hydroxyprostaglandin dehydrogenase is down-regulated in gastric cancer. Clin. Cancer Res. 15, 4572.

Thill, M., Fischer, D., Kelling, K., Hoellen, F., Dittmer, C., Hornemann, A., Salehin, D., Diedrich, K., Friedrich, M., and Becker, S. (2010). Expression of vitamin D receptor (VDR), cyclooxygenase-2 (COX-2) and 15-hydroxyprostaglandin dehydrogenase (15-PGDH) in benign and malignant ovarian tissue and 25-hydroxycholecalciferol (25 (OH2) D3) and prostaglandin E2 (PGE2) serum level in ovarian cancer patients. J. Stero. Biochem. Mol. Biol. 121, 387-390.

van der Flier, L., van Gijn, M., Hatzis, P., Kujala, P., Haegebarth, A., Stange, D., Begthel, H., van den Born, M., Guryev, V., and Oving, I. (2009). Transcription factor achaete scute-like 2 controls intestinal stem cell fate. Cell 136, 903-912.

Wang, X., Chorley, B.N., Pittman, G.S., Kleeberger, S.R., Brothers, J. 2nd., Liu, G., Spira, A., and Bell, DA. (2010). Genetic variation and antioxidant response gene expression in the bronchial airway epithelium of smokers at risk for lung cancer. PloS One 5, e11934.

Wu, C., Tseng, R., Hsu, H., Wang, Y., and Hsu, M. (2009). Frequent down-regulation of hRAB37 in metastatic tumor by genetic and epigenetic mechanisms in lung cancer. Lung Cancer 63, 360-367. 